\title{
All-Optical Decision-Gating of 10-Gb/s RZ Data in a Semiconductor Optical Amplifier Temporally Gain-Shaped With Dark-Optical-Comb
}

\author{
Gong-Ru Lin, Senior Member, IEEE, Kun-Chieh Yu, Ci-Ling Pan, and Yu-Sheng Liao
}

\begin{abstract}
We demonstrate a novel all-optical noninverted OC-192 return-to-zero (RZ) decision-gate by using a semiconductor optical amplifier (SOA) which is gain-controlled to achieve an extremely high cross-gain-modulation depth and a narrow gain window. A dark-optical-comb generated by reshaping the optical clock RZ data in a Mach-Zehnder intensity modulator is employed as an injecting source to temporally deplete most of the gain in the SOA. Such a dark-optical-comb injected SOA decision-gate exhibits improved $3 R$ regeneration performances such as a timing tolerance of $33.5 \mathrm{ps,} \mathrm{a} Q$-factor of 8.1 , an input dynamical tolerance of $14 \mathrm{~dB}$, and an extinction ratio (ER) of $14 \mathrm{~dB}$. The deviation between the wavelengths of backward injected dark-optical-comb and input $R Z$ data for optimizing the ER of the decision-gate is determined as $\Delta \lambda=19 \mathrm{~nm}$. Under a threshold operating dark-optical-comb power of $7 \mathrm{dBm}$, such a decision-gate can recover the $-18.5-\mathrm{dBm}$ degraded $\mathrm{RZ}$ data with a bit-error-rate of less than $10^{-9}$ at $10 \mathrm{~Gb} / \mathrm{s}$. A negative power penalty of $-4.2 \mathrm{~dB}$ is demonstrated for the $\mathrm{RZ}$ data after $50-\mathrm{km}$ propagation and decision gating.
\end{abstract}

Index Terms-All optical, decision gate, gain shaping, injection, OC-192, optical comb, return to zero (RZ), semiconductor optical amplifier (SOA).

\section{INTRODUCTION}

$\mathbf{I}$ $\mathrm{N}$ HIGH-SPEED and broadband optical-time-divisionmultiplexing (OTDM) communication networks, the optical return-to-zero (RZ) data pattern is always distorted during longhaul fiber-optic transmission, in which the optical RZ data also experiences severe degradation on signal-to-noise ratio (SNR), extinction ratio (ER), timing jitter, and bit-error-rate (BER). The reamplifying, retiming, and reshaping ( $3 R$ ) regeneration of the optical RZ data is, therefore, essential to extend the reach of long-haul transmission with a large capacity and to restore the impairments imposed at the OTDM-wavelength-divisionmultiplexing interface. To meet this demand, several all-optical approaches based on semiconductor optical amplifiers (SOAs) [1]-[3] have been proposed to be competitive with those han-

Manuscript received November 27, 2006; revised March 16, 2007. This work was supported in part by National Science Council under Grants NSC95-2221E-002-448 and NSC96-2752-E-009-008-PAE.

G.-R. Lin is with the Graduate Institute of Electro-Optical Engineering and the Department of Electrical Engineering, National Taiwan University, Taipei 106, Taiwan, R.O.C. (e-mail: grlin@ @ntu.edu.tw).

K.-C. Yu, C.-L. Pan and Y.-S. Liao are with the Department of Photonics and Institute of Electro-Optical Engineering, National Chiao Tung University, Hsinchu 300, Taiwan, R.O.C.

Color versions of one or more of the figures in this paper are available online at http://ieeexplore.ieee.org.

Digital Object Identifier 10.1109/JLT.2007.897722 dled by electronics, in which the maximum data rate of the alloptical 3R regeneration has been increased to $80 \mathrm{~Gb} / \mathrm{s}$ [4].

Alternatively, all-optical decision-gating has also emerged as a simplified technology with reamplifying and reshaping functions for all-optical 3R system to obtain regenerated optical RZ data. The all-optical decision-gate-based optical repeaters are essential components for recovering the degraded optical RZ data in photonic switching systems or OTDM networks. In principle, an optical RZ data and an optical clock are simultaneously coupled into such an optical AND gate to regenerate (including $3 \mathrm{R}$ procedures) the input and degraded optical signal. Several all-optical decision-gates made for the purpose of alloptical 3R regeneration have emerged during the past decade, while some approaches were demonstrated at bit rate even up to $40 \mathrm{~Gb} / \mathrm{s}$. Experimentally, the decision-gating is achieved by temporally confining a window with versatile techniques such as SOA-based cross-gain [5] or cross-phase modulation (XGM or XPM), SOA-integrated Michelson/Mach-Zehnder [6], [7] or optical loop mirror interferometry [8], and four-wave mixing (FWM) [9]. Versatile optical decision-gating schemes, particularly the cross-gain-modulated SOA-based all-optical RZ decision-gate have recently been studied [10]. The degraded data stream is eventually reshaped by the clock stream in the SOA to regenerate an RZ data stream with a better data pattern, smaller noise and timing jitter, and improved SNR. However, up to four SOAs were employed in previous approach to recover the clock, to reduce the amplitude fluctuation, and to decision-gate the RZ data [11]. Recently, we have proposed a dark-optical-comb injection technique for mode-locking the SOA-based fiber laser [12], [13] and for converting the format of NRZ data stream in the SOA [14]. In this paper, we demonstrate for the first time a novel all-optical RZ decisiongating by using an optically gain controlled SOA, which is backward injected by a dark-optical-comb generated from the received optical clock. The gain window of the SOA is greatly narrowed by backward injecting the dark-optical-comb, which is a reshaped optical clock with an extremely large duty cycle after passing through a comb-driven Mach-Zehnder intensity modulator (MZM).

\section{EXPERIMENTAL SETUP}

The experimental setup of the SOA-based all-optical decision-gate is shown in Fig. 1, which consists of an SOA (QPhotonics LLC, QSOA-1550), a distributed feedback laser diode, an optical clock data recovery, an electrical comb 


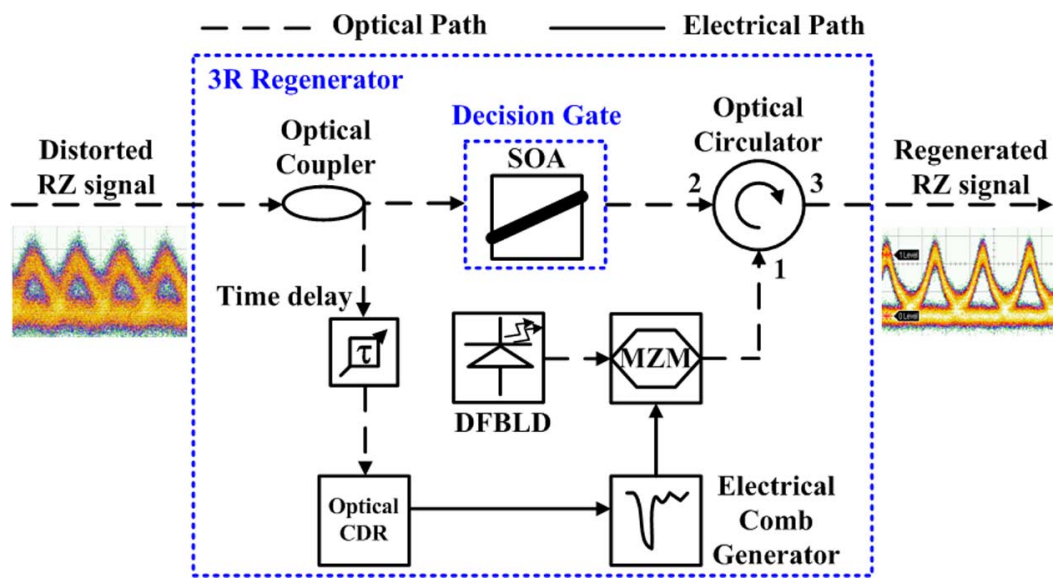

Fig. 1. Schematic diagram of the backward optical-comb injection SOA-based decision-gate.

generator, an MZM, and an optical circulator. Part of the received optical RZ signal is clock recovered and reshaped to inject the SOA for temporal gain depletion. In experiment, a $10-\mathrm{Gb} / \mathrm{s}$ optical degraded RZ pseudorandom binary sequence (PRBS) data with pattern length of $2^{23}-1$ and output power from -10 to $-1 \mathrm{dBm}$ is employed as the incoming data stream. The SOA with a gain peak at $1530 \mathrm{~nm}$ and an amplified spontaneous emission linewidth of $35 \mathrm{~nm}$ is operated at $280 \mathrm{~mA}$ (high-gain condition). The small-signal gain and typical output power of the SOA at nearly saturating condition are $20 \mathrm{~dB}$ and $15 \mathrm{~mW}$, respectively. The dark-optical-comb pulsetrain is generated by optically reshaping the received clock signal with an MZM driving by an electrical comb generator. A dark-optical-comb with average power, pulsewidth, and a large duty cycle of $4.46 \mathrm{~mW}, \leq 60 \mathrm{ps}$, and $78 \%$, respectively, can be obtained at the MZM output by changing the dc-bias level of the MZM at the nonlinear region. To temporally gain-deplete the SOA at highly biased condition, the dark-optical-comb is then amplified from 7 to $18 \mathrm{dBm}$ and backward-injected into the SOA via an optical circulator. The RZ data pattern is analyzed by a digitized sampling oscilloscope (Agilent, 86100+86109A) and a BER detector (Agilent, 71612C).

\section{PRinCIPLE AND Simulation}

The amplification in an SOA of an input signal depletes the carrier density and reduces gain, which causes a shift of spectrum and a change of carrier lifetime. The ER, which is the main figure-of-merit for a reshaped data-stream, is determined by taking into account both the gain depletion and gain-spectral shifting effects occurring in the SOA under high-power injection. The gain model of two-level system in SOA as having a Lorentzian line shape as given in [15]

$$
g_{\lambda, N}=\frac{a\left(N-N_{0}\right)}{1+\left(\lambda-\lambda_{N}\right)^{2} /\left(\Delta \lambda_{g}\right)^{2}}
$$

where $N$ is the carrier density, $a$ is the differential gain, $\lambda_{N}$ is the peak wavelength at carrier density $N, \lambda_{0}$ is the peak wavelength at transparency, and $\Delta \lambda_{g}$ is the $3-\mathrm{dB}$ bandwidth

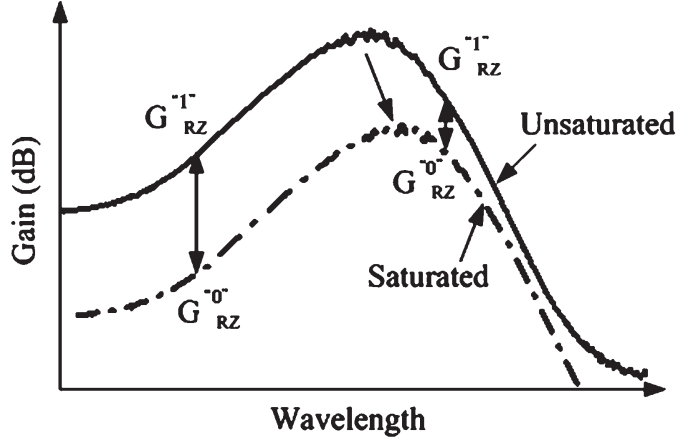

Fig. 2. SOA gain shift under saturation conditions causing a smaller ER at longer wavelengths and a larger ER at shorter wavelengths.

of the gain coefficient. Such a spectral shift in the SOA can be realized by the expression of

$$
\lambda_{N}=\lambda_{0}-\kappa_{0}\left(N-N_{0}\right)
$$

where $\lambda_{0}$ is the peak wavelength at transparency, and $\kappa_{0}$ is a constant characterizing the gain-peak shift. Under saturated conditions, the peak wavelength of the SOA gain has shifted from 1530 to $1550 \mathrm{~nm}$ under the strong backward injection at $1550 \mathrm{~nm}$, as shown in Fig. 2. The shift of SOA gain peak to a longer backward injecting wavelength is mainly attributed due to the depletion of carriers in the SOA by the intense opticalclock injection. The gain of the SOA cannot be fully depleted if the dark-optical-comb injects into the SOA at wavelength nearly the original gain peak (i.e., backward injection at shorter wavelength). Since such a short-wavelength injection only triggers the stimulated emission of conduction-band carriers at higher energy states, which thus consumes carriers upon higher energy levels and leaves other carriers below the injecting band undepleted. Then, the undepleted carriers will contribute to an upward offset " 0 " level of the regenerated signal and cause a small ER. It is preferred to locate the wavelength near but slightly longer than the gain peak, as the gain SOA can be depleted more severely at a larger gain region and, thus, red-shifted to a longer wavelength. However, the depletion of carriers, as well as gain of the SOA, becomes less significant if a backward injecting wavelength is too long to exceed the 


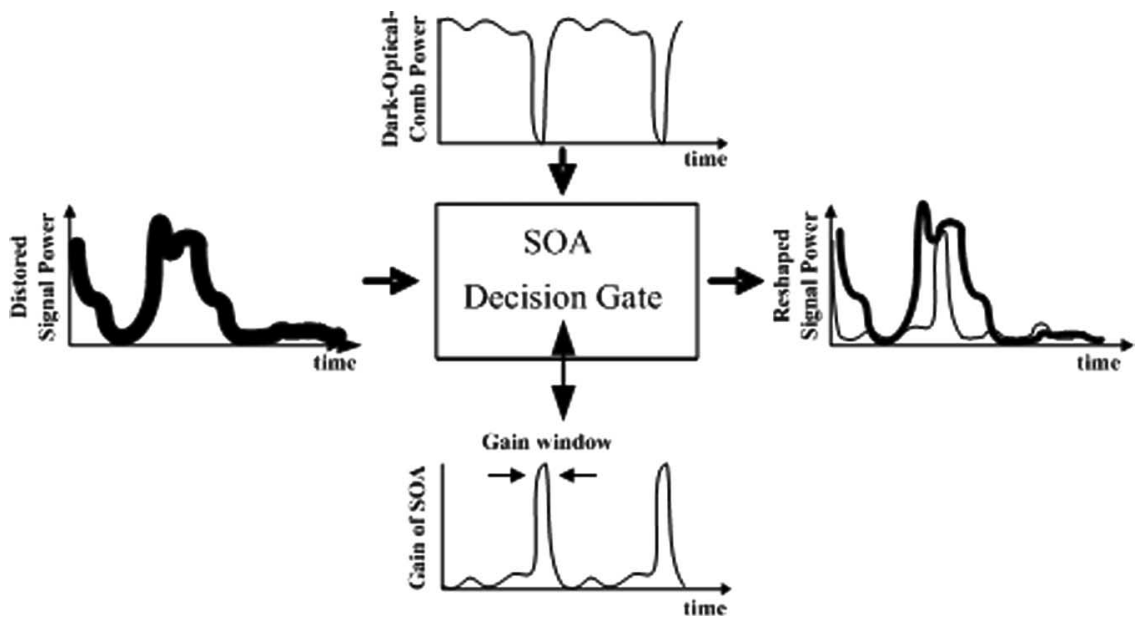

Fig. 3. Operating principle of the reshaped optical-clock controlled SOA RZ decision gate.
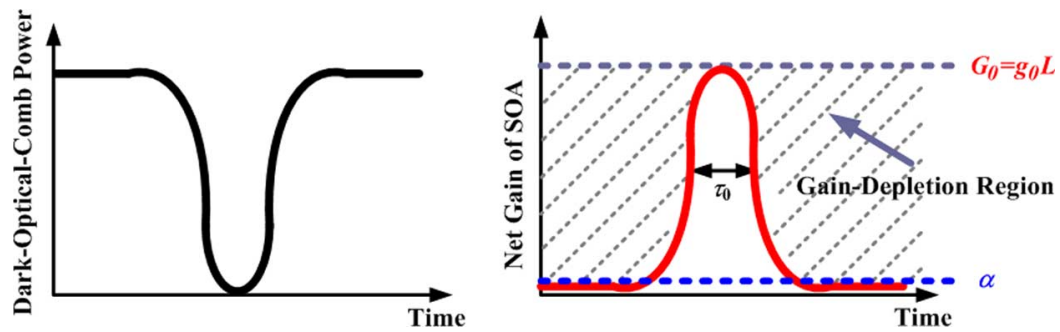

Fig. 4. Temporally reshaped gain window of the (right) SOA decision gate under the backward injection of (left) the dark-optical-comb.

original gain peak, which in turn causes a reduction in ER of the input data-stream at shorter wavelengths.

On the other hand, the use of the high-level electrical pumping accompanied with a strong optical injection to concurrently suppress lasing and enhance carrier/photon interaction in the SOA can significantly result in a transforming speed of larger than the carrier recovery rate of the SOA. The speed limitation for the SOA-based data pattern transformer is determined by the gain recovery time of the SOA. The gain recovery time $\tau$ in the SOA is described as

$$
\tau^{-1}=\tau_{\mathrm{nr}}^{-1}+\boldsymbol{a} \boldsymbol{S}
$$

where $\tau_{\mathrm{nr}}$ is the nonradiative recombination time, $\boldsymbol{a}$ is the stimulated emission rate, and $\boldsymbol{S}$ is the internal photon density in the SOA. It is elucidated that the gain recovery time of the SOA, as well as the rising time of the transformed pulse data pattern, can be effectively shortened by greatly increasing the internal photon density of the SOA after strong backward injection. The backward injection of the dark-optical-comb with extremely large duty-cycle thus results in an ultranarrow gain window with enhanced switching response, facilitating the convertible data rate up to $10 \mathrm{~Gb} / \mathrm{s}$ and beyond. In contrast, the optical gain of the SOA in our case is relatively complicated, as compared to the amplifier gain obtained under typical operation of the SOA. The gain window of the SOA is temporally modified to implement $3 \mathrm{R}$ of the incoming RZ data-stream with distorted shape, as shown in Fig. 3.

Theoretically, the transfer function of transient gain for the SOA under backward dark-optical-comb injection can be deduced by modifying the similar model [16]. It is necessary to model pulse propagation through the temporally gain-depleted SOA with a traveling-wave equation containing spatial and time derivatives. Assume that the backward dark-optical-comb injection function with a form of $P_{\text {in }}(\tau)=P_{0}\left[1-P^{\prime}(\tau)\right]$, where $P^{\prime}(\tau)$ exhibits a Gaussian pulse shape. That is

$$
P_{\text {in }}(\tau)=\frac{E_{\text {in }}}{\tau_{0} \sqrt{\pi}}\left[1-\exp \left(-\frac{\tau^{2}}{\tau_{0}^{2}}\right)\right]
$$

where $E_{\text {in }}$ is the pulse energy, and $\tau_{0}$ is the full-width at half-maximum of the dark-optical-comb pulse. The first-term on right-handed side (RHS) is used to complete deplete the SOA gain by inducing stimulated emission at the injecting wavelength, and the second-term leaves the residual gain window of the SOA with a very short duty-cycle within one period. This input power function precisely describes the waveform of a backward injected dark-optical-comb before entering the SOA.

With an appropriate backward injecting power, the darkoptical-comb (i.e., an inverse Gaussian shape with a small duty cycle in one period) can fully deplete most of the SOA gain within one period, as illustrated in Fig. 4. This eventually leads to a narrowing effect on the residual gain window of the SOA, which remains only a Gaussian shape with a small duty cycle. In our case, the rate equation of carrier density can be transferred into a rate equation for gain that is described as [16]

$$
\frac{\partial g}{\partial \tau}=\frac{\left(g-g_{0}\right)}{\tau_{c}}-\frac{g P_{\mathrm{in}}}{E_{\mathrm{sat}}}
$$




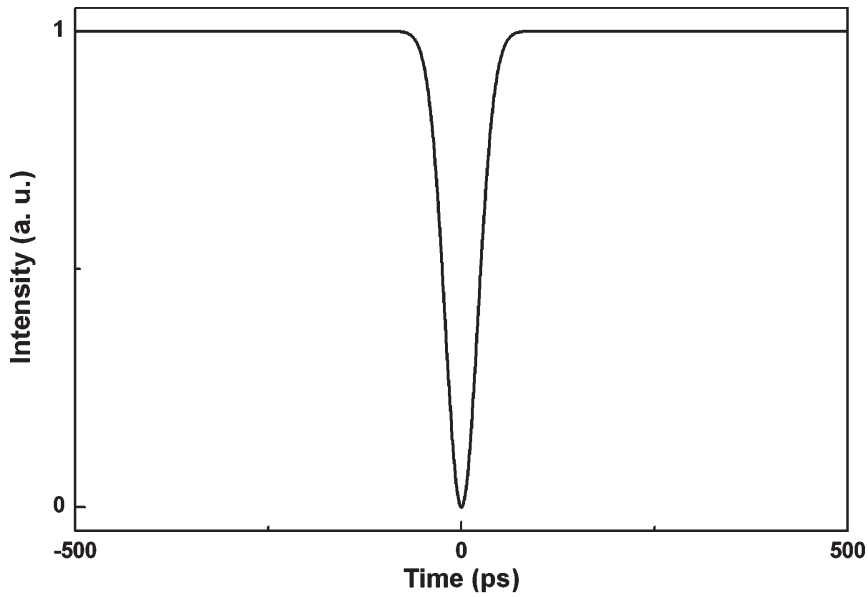

Fig. 5. Simulation of one-period dark-optical-comb signal.

where $g_{0}, \tau_{c}$, and $E_{\text {sat }}$ are the small signal gain coefficient, carrier lifetime, and saturation energy for the amplifier, respectively. Solving (5) will lead to

$$
G(\tau)=\exp [h(\tau)]=\exp \left[\int_{0}^{\tau} g(z, \tau) d z\right]
$$

with $h(\tau)$ denoting the integrated gain and $G(\tau)$ denoting the transfer function of the instantaneous gain of SOA under backward dark-optical-comb injection with an inverse Gaussian like waveform. Integrating (5) and using (6) gives an ordinary differential equation for $h$

$\frac{d h}{d \tau}=\left(\frac{g_{0} L-h}{\tau_{c}}-\frac{E_{\mathrm{in}}}{\tau_{0} \sqrt{\pi} E_{\mathrm{sat}}}\right)+\frac{E_{\mathrm{in}}}{\tau_{0} \sqrt{\pi} E_{\mathrm{sat}}} \exp \left(-\frac{\tau^{2}}{\tau_{0}^{2}}\right)$.

With the backward injection of an inversed Gaussian-like function $h(\tau)$ shown in Fig. 5, the original SOA gain can almost be depleted in one period. That is, the first-term on the RHS of (7) can be neglected by canceling each other. Concurrently, the residual gain becomes the inverse shape of the incident waveform with its coefficient equivalent to the maximum gain of the SOA. Therefore, we can simplify and rewrite (7) as

$$
\frac{d h}{d \tau}=\frac{g_{0} L}{\tau_{c}} \exp \left(-\frac{\tau^{2}}{\tau_{0}^{2}}\right)-\frac{h}{\tau_{c}}
$$

and (8) can be solved to obtain $h(\tau)$ for a given input inverse pulse shape and gain $g_{0} L$. The solution of (8) is

$$
h(\tau)=-\frac{g_{0} L \tau_{0} \sqrt{\pi}}{2 \tau_{c}} e^{\frac{\tau_{0}^{2}}{4 \tau_{c}^{2}}-\frac{\tau}{\tau_{c}}} \operatorname{erf}\left(\frac{\tau_{0}}{2 \tau_{c}}-\frac{\tau}{\tau_{0}}\right)
$$

where erf is the error function

$$
\begin{aligned}
G(\tau) & =\exp [h(\tau)] \\
& =\exp \left\{-\frac{g_{0} L \tau_{0} \sqrt{\pi}}{2 \tau_{c}} e^{\frac{\tau_{0}^{2}}{4 \tau_{c}^{2}}-\frac{\tau}{\tau_{c}}} \operatorname{erf}\left(\frac{\tau_{0}}{2 \tau_{c}}-\frac{\tau}{\tau_{0}}\right)\right\} .
\end{aligned}
$$

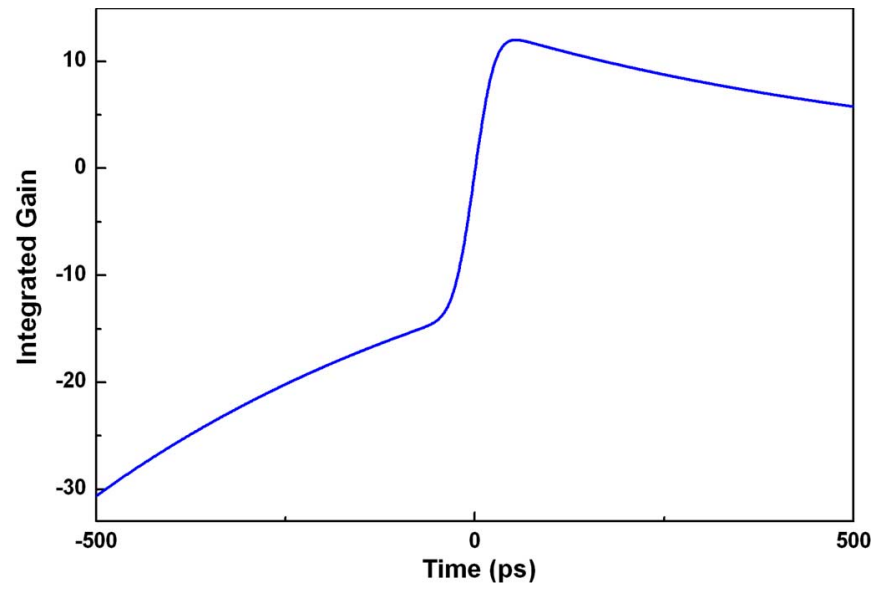

Fig. 6. Simulation of integrated gain $h(\tau)$ in the SOA by dark-optical-comb injection.

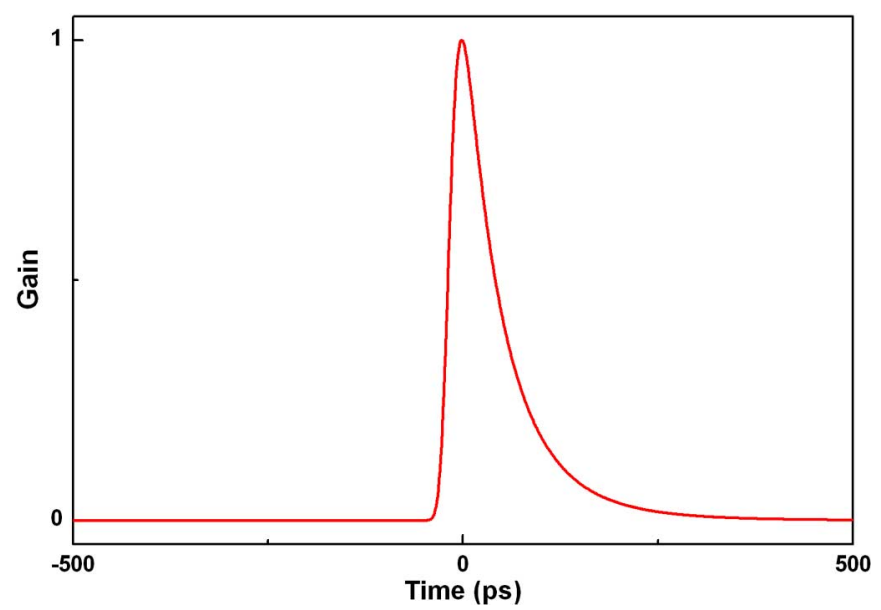

Fig. 7. Simulation of instantaneous gain $G(\tau)$ in the SOA by dark-opticalcomb injection.

As a result, the simulations of the injected dark-opticalcomb and the reshaped $h(\tau)$ and $G(\tau)$ of the backward dark-optical-comb injected SOA are shown in Figs. 5-7, respectively.

The normalized on-level of the input power function shown in Fig. 5 first reduces the SOA gain to below the transparent (i.e., loss) condition. Subsequently, the SOA gain is built up again within the instantaneous off-level of the power function, providing a short rising-edge but long trailing-edge smallsignal gain function. Such an operation eventually reshapes the continuous-wave gain of the SOA into a greatly shortened gain window, as shown in Fig. 7. Our simulation reveals that the temporal shrinkage in the gain window of the SOA is mandatory to optimize the regeneration of the decisiongated data.

\section{RESUlts AND Discussions}

In principle, shrinking the gain window of the SOA in the time domain is mandatory to optimize the RZ decision-gating in our proposed scheme. As shown in Fig. 3, the optimized decision-gating of the input data pattern can be achieved under 


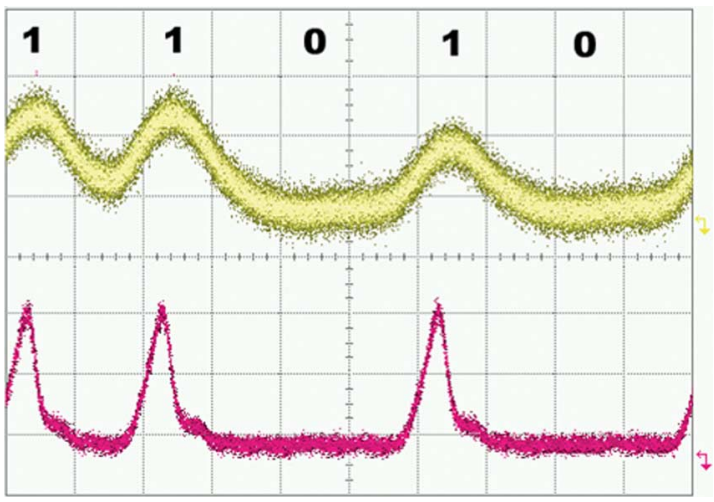

Fig. 8. (Top) Distorted RZ data-stream with "11010" pattern at $10 \mathrm{~Gb} / \mathrm{s}$. (Bottom) Converted data stream.
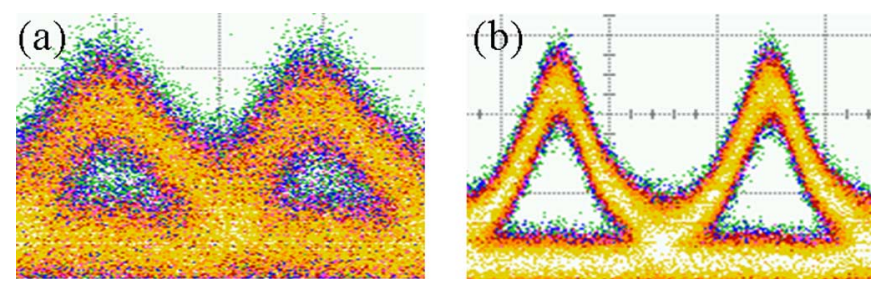

Fig. 9. Eye diagrams of (a) distorted input RZ signal and (b) decision-gated RZ signal.

serious gain depletion in the SOA for strong gain-window narrowing. This can only be implemented by backward injecting the SOA with an extremely large-duty-cycle signal. For example, an RZ data stream (11010) encounters the residual gain in the SOA and transforms into a pulse pattern, as shown in Fig. 8. The eye diagrams at the input and after our proposed $3 \mathrm{R}$ regenerator are shown in Fig. 9. As the injected optically dark-optical-comb power is turned off (i.e., at a low injected level), the gain experienced by the input data is increased, and the gain recovery time is shortened by the bit " 1 " of the probe data.

The regenerated eye is very clean and has a slightly reduced pulsewidth ( $28 \mathrm{ps}$ ) due to the shape of the gating window. The amplitude margin defined by the voltage decision level can be used in BER evaluation by measuring the SNR (or $Q$-factor) in decision circuits. Previously, Bergano et al. have demonstrated a BER evaluation method by measuring the SNR at decision circuit of an optical transmission and receiving system [17], [18], which is given by $Q=\left(I_{1}-I_{0}\right) /\left(\sigma_{1}+\sigma_{0}\right)$ and $\mathrm{BER}=0.5 \cdot \operatorname{erfc}(Q / \sqrt{ } 2)$, where $I_{1,0}$ and $\sigma_{1,0}$ are the mean value and standard deviation of the mark and space data rail, and $\operatorname{erfc}(x)$ is the complementary error function. The measured BER of the data stream can be accurately calculated from the recorded $Q$-factor of the received eye pattern at a desired data rate. The equivalent mean and sigma of the marks and spaces are determined by fitting this data to the Gaussian characteristic. The measured $Q$-factor of the decision-gated RZ data can be improved from 6.0 to 8.1, providing an improved BER from $1 \times 10^{-9}$ to $2 \times 10^{-16}$ at an RZ data rate of $10 \mathrm{~Gb} / \mathrm{s}$.

On the other hand, the noise reduction of the decision-gated data stream is mainly attributed to the gain-saturated SOA with a slightly reduced gating window of only $33.5 \mathrm{ps}$. It can further be calculated that under a high level accompanied with

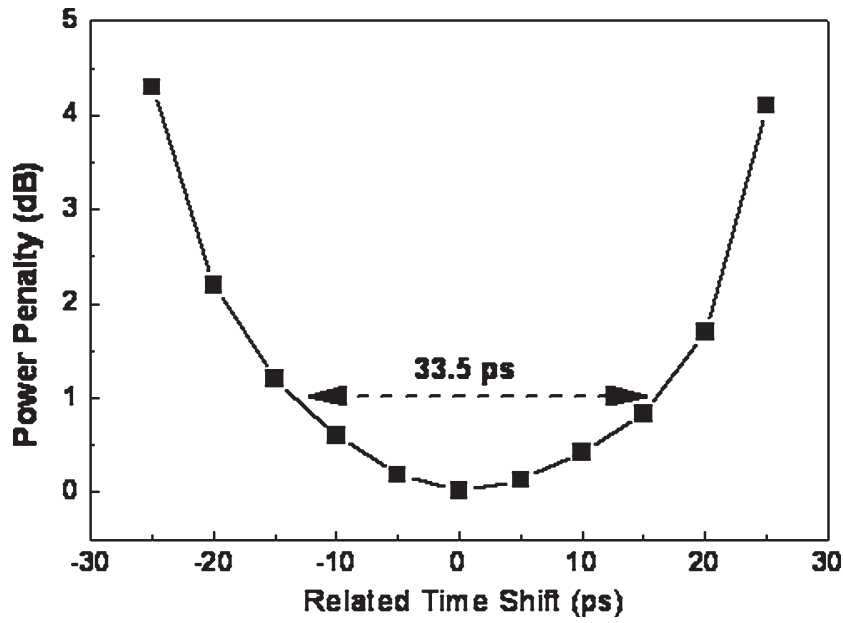

Fig. 10. Timing tolerance of the SOA-based all-optical RZ decision gate.

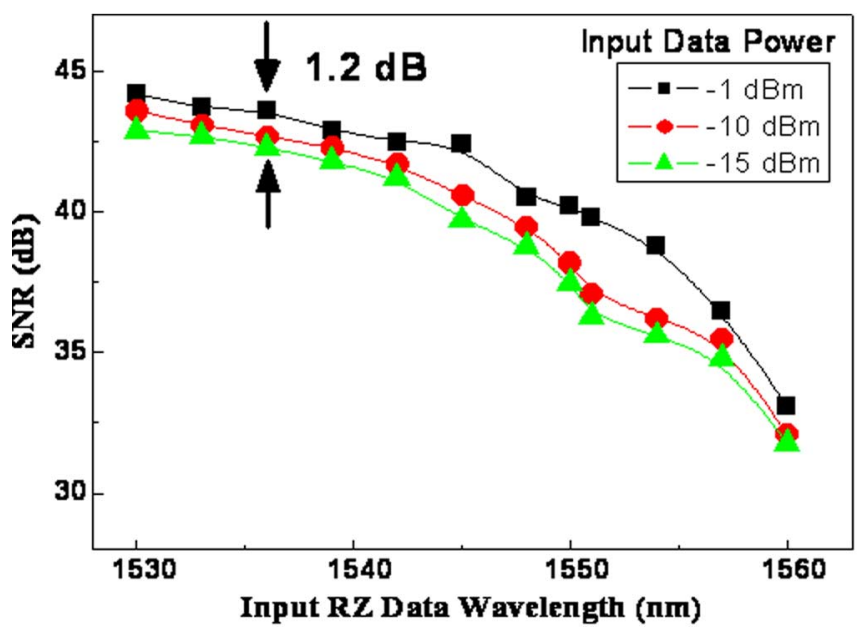

Fig. 11. SNR of the decision-gated RZ signal as a function of RZ data wavelength at different powers.

an intense optical injection, the SOA is forced to suppress its stimulated emission and enhance carrier/photon interactions, thus providing a conversion speed of faster than the carrier recovery rate. In our case, the increase on both of the data and backward injection power also result in the shortened rising and falling time of decision-gated RZ signals, respectively. The timing tolerance for initiating the all-optical decision-gating function in the SOA is evaluated by shifting the phase of the clock-recovered 10-GHz dark-combed signal before injecting into the SOA, which changes the relative time delay between the degraded RZ data stream and the gating window.

As the result, the timing tolerance on the BER of the decision-gated $\mathrm{RZ}$ data within $1-\mathrm{dB}$ power penalty up to 33.5 ps is allowed, as shown in Fig. 10. In principle, the all-optical gating in the backward dark-optical-comb injected SOA with a larger SNR can be obtained setting the incoming RZ data at a shorter wavelengths. Fig. 11 shows the SNR of the regenerated RZ signal versus the wavelength of the distorted RZ signal at the input optical powers of $-1,-10$, and $-15 \mathrm{dBm}$. As the wavelength of the input RZ data is far from that of the backward injected dark-optical-comb, the crosstalk between signal and the controlled optical clock (i.e., 

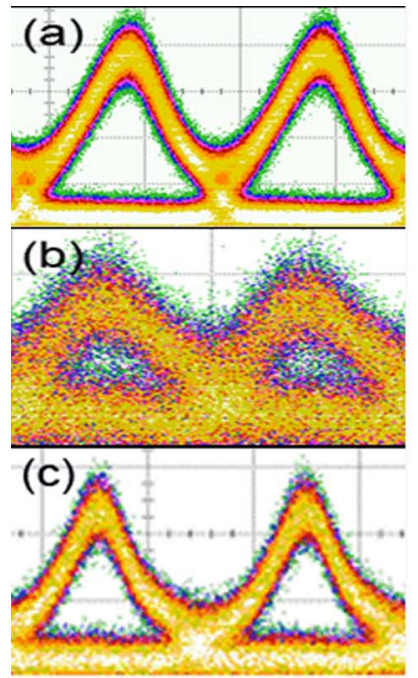

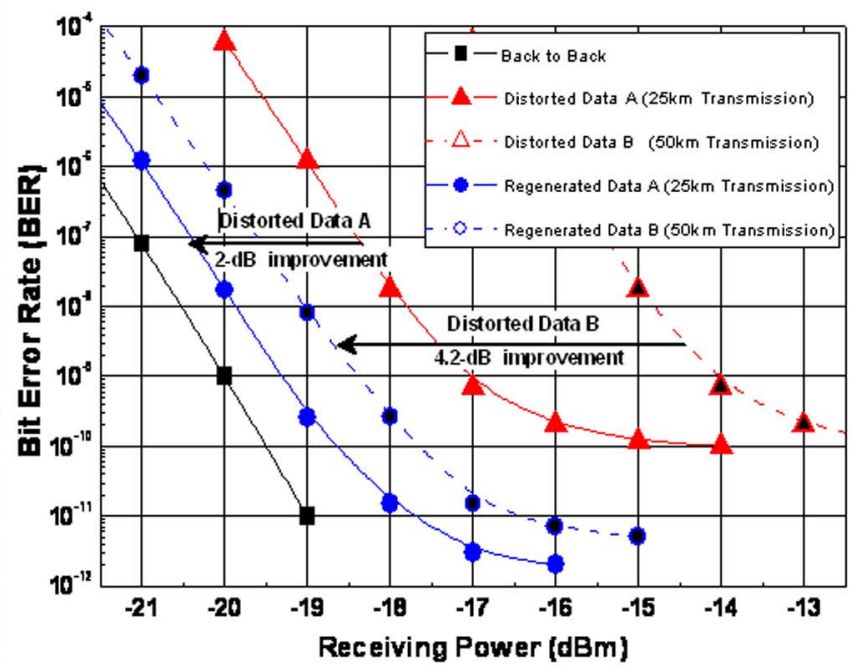

Fig. 12. (Left) Eye diagrams of the (a) back-to-back, (b) distorted, and (c) regenerated RZ PRBS data-streams at 10 Gb/s. (Right) BER analysis the RZ data stream after back-to-back transmission (black solid square), propagation through SMF-28 spool of $25 \mathrm{~km}$ (blue solid circle) and $50 \mathrm{~km}$ (red solid triangle), and those decision-gated by the dark-optical-comb gain-reshaped SOA (blue hollow circle and red hollow triangle), respectively.

the dark-optical-comb) can effectively be reduced, providing an output RZ data stream with relatively higher SNR. Identical to XGM wavelength conversion, this scheme has the potential advantages of high operation speed, simple implementation, larger dynamical range, and ease of use. Shrinking the gain window of the SOA in the time domain is mandatory to optimize the optical decision gate. Only 1.2-dB degradation on SNR of the decision-gated RZ data is observed.

In our experimental setup, the distorted RZ data stream was made by propagating through SMF-28 fiber spools with lengths of 25 and $50 \mathrm{~km}$. Such chirp-uncompensated RZ data streams experience serious dispersion to degrade its shape during propagation, as shown in Fig. 12(a). The dispersion of SMF simultaneously broadens the RZ data stream and degrades the performance of amplitude noise and timing jitter, as shown in Fig. 12(b). The BER of two distorted data-streams and their regenerated data-streams at $10 \mathrm{~Gb} / \mathrm{s}$ are measured as a function of received data power, as shown in right part of Fig. 12. In the SOA-based all-optical decision-gator, the injected dark-optical-comb pulse-train results in an improvement of conversion speed, which effectively reduces the rise time of the converted signal [19]. The original sensitivity of the backto-back transmitted RZ data at a BER $<10^{-12}$ is as low as $-19.5 \mathrm{dBm}$. After a $25-\mathrm{km}$ transmission without any dispersion compensation, the RZ data stream is greatly distorted with SNR as small as 3 . The eye diagram of the distorted signal is consequently broadened and distorted, leading to the receiving power penalties of the optical RZ signals further degraded by 2.7 and $5.4 \mathrm{~dB}$ after propagating through $25-$ and $50-\mathrm{km}$ transmission, respectively. In contrast, the regenerated RZ signal is well shaped with a recovered SNR after decision gating by the backward dark-optical-comb injected SOA, providing a reduced power penalties as low as 0.7 and $1.2 \mathrm{~dB}$ with $25-$ and 50-km transmission. Note that there are error floors shown in the BER analysis of both the propagated and decision-gated RZ data, which could be removed by further suppressing amplified spontaneous noise floor of the SOA with a ultranarrow band-

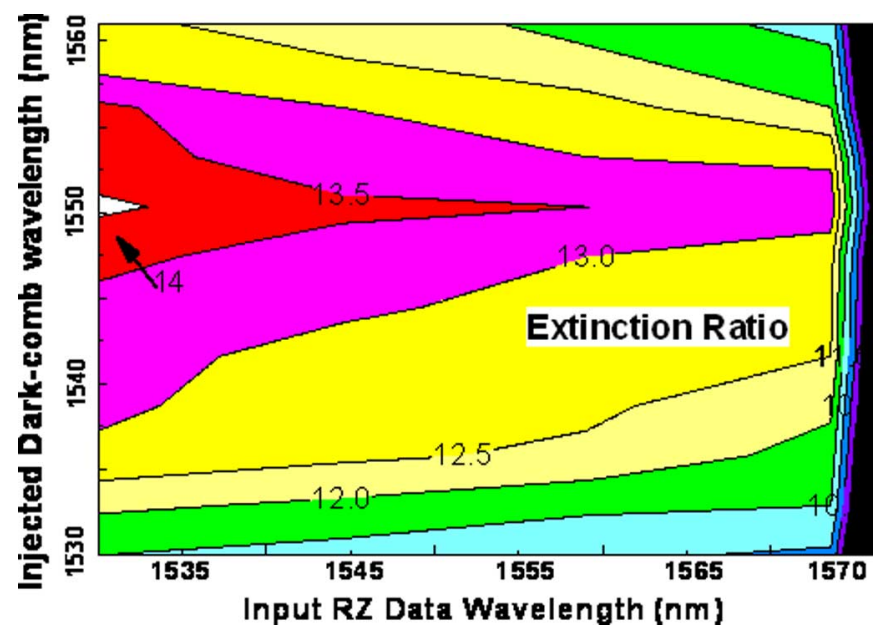

Fig. 13. Contour plot of the ER with dark-optical-comb wavelength and input data wavelength.

pass filter with a linewidth of $<0.3 \mathrm{~nm}$. In brief, a negative power penalty of up to $-4.2 \mathrm{~dB}$ is obtained for the $50-\mathrm{km}$ propagated RZ data after decision-gating.

Most important, the dependence between the input data/ injection wavelength and the ER of the output RZ signal has also been elucidated. Fig. 13 shows the contour plot for the ER of the output RZ data as a function of the injected darkoptical-comb and the input data wavelengths. It is observed from experimental results that the ER of the output RZ data is determined by taking into account both the gain depletion and the gain-spectral shifting effects occurring in the SOA under high-power injection, which increases for shorter wavelength, indicating that shorter data wavelength accompanied with a long-wavelength injection is essentially preferred for the decision gating of the RZ data in the SOA. The deep red region of Fig. 13 indicates the optimized wavelength setting region for the SOA-based decision-gate with the highest ER. The data wavelength set at the gain peak of the SOA for the highest gain is not at $1530 \mathrm{~nm}$, but the injected optical-clock has to be shifted 


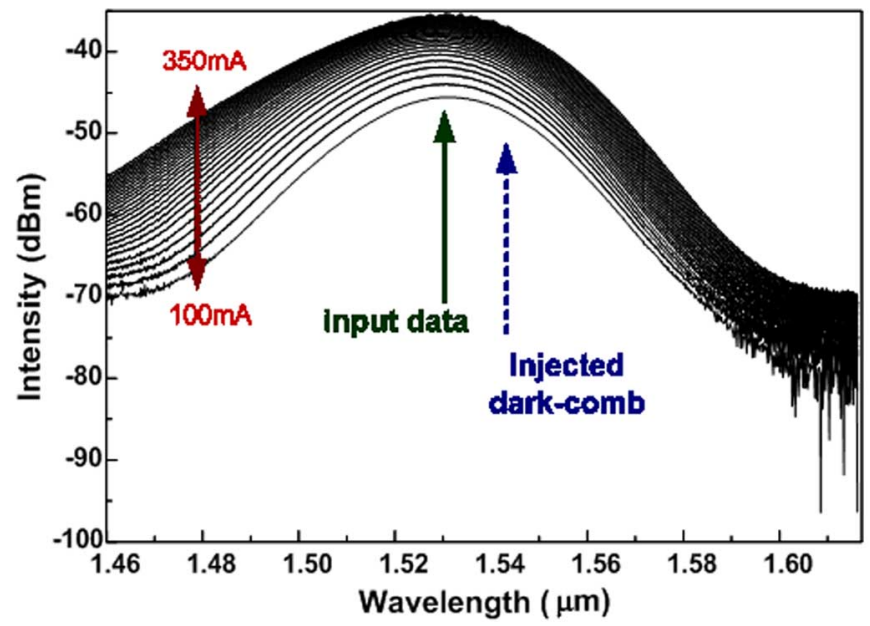

Fig. 14. ASE spectrum of this SOA at different biasing currents.

to $1550 \mathrm{~nm}$ for the largest gain depletion of the SOA at the time interval between data bits, which is due to the depletion of carriers by the intense injected optical-clock [15].

The gain SOA can be depleted more severely at longer wavelength, which eventually leads to a higher ER of the input RZ data-stream at shorter wavelengths as the gain of the SOA is changing from the saturation to the depletion condition. This results in the gain peak of ASE and the optimal dark-opticalcomb wavelength being shifted to a longer wavelength due to the depletion of carriers, as shown in Fig. 14. It is preferred to locate the injected optical-clock at wavelengths near but slightly longer than the gain peak, as the gain SOA can be depleted more severely at larger gain region and, thus, red-shifted to a longer wavelength. The ER (i.e., power ratio of the transmitted "1" data to the transmitted " 0 " data) of the incoming distorted RZ PBRS data is about $7 \mathrm{~dB}$. In the optimum region, the measured eye diagram for the RZ PRBS data reveals a greatly improved ER of $14 \mathrm{~dB}$.

It is worth comparing the proposed approach with current architectures employing same device and different techniques. Under a sinusoidal optical clock injection, the conventional XGM scheme usually exhibits a limited pulsewidth and a worse ER due to insufficient gain depletion window under sinusoidal-clock injection, whereas the XPM and interferometric approaches strictly relies on the rigorous control of the injection power and the timing delay between the data and the clock stream. FWM is a wavelength- and polarizationsensitive technique against most unpolarized optical networks. Except for the XGM approach, the bulky configuration, precisely delay-time control, and specially designed waveguide components of the aforementioned architectures are detrimental to versatile applications. In contrast, our concept adopts the typical XGM system by injecting a dark-optical-comb to cause an extremely narrowed gain window with a duty cycle of less than $30 \%$, which greatly reduces the timing jitter of input data in the SOA to $<2.5$ ps. Moreover, such intensity further reduces the rise/fall time and thus improves the effective modulation bandwidth of the SOA, which essentially facilitates the operation of RZ decision-gating at higher data rates. The ON/OFF ER can be enhanced up to $15 \mathrm{~dB}$ by taking into ac-
TABLE I

PARAMETRIC COMPARISON OF DIFFERENT SOA-BASED RZ Decision-Gates. $P$ : Power Penalty, $\Delta \lambda$ : Wavelength Preserving CAPABILITY, $\Delta \tau / \tau$ : DUTY-CYCle OF DeCisION-Gated RZ, $R$ : ON/OFF ER OF DECISION-GATED RZ, $\Delta P$ : DYNAMIC RANGE OF THE INPUT, AND $\Delta T$ : Timing TOLERANCE IN UNIT OF DUty CyCLE BETWEEN INPUT AND INJECTED CLOCK

\begin{tabular}{ccccccc}
\hline \hline & $\mathrm{P}$ & $\Delta \lambda$ & $\Delta \tau / \tau$ & $\mathrm{R}$ & $\Delta \mathrm{P}$ & $\Delta \mathrm{T}$ \\
\hline XGM-SOA $^{[5]}$ & $-3.5 \mathrm{~dB}$ & $0 \mathrm{~nm}$ & $50 \%$ & - & - & - \\
MI- SOA $^{[6]}$ & $1 \mathrm{~dB}$ & $10 \mathrm{~nm}$ & $25 \%$ & $10 \mathrm{~dB}$ & - & $12 \%$ \\
LOM-SOA $^{[8]}$ & - & $10 \mathrm{~nm}$ & - & - & $10 \mathrm{~dB}$ & $10 \%$ \\
MZI-SOA $^{[7]}$ & $1.5 \mathrm{~dB}$ & $0 \mathrm{~nm}$ & $50 \%$ & - & - & - \\
FWM-SOA $^{[9]}$ & $1 \mathrm{~dB}$ & $10 \mathrm{~nm}$ & $25 \%$ & $19 \mathrm{~dB}$ & - & - \\
DOC-XGM-SOA $^{-4.2 \mathrm{~dB}}$ & $0 \mathrm{~nm}$ & $28 \%$ & $14 \mathrm{~dB}$ & $14 \mathrm{~dB}$ & $34 \%$ \\
\hline \hline
\end{tabular}

count both the gain depletion and gain-spectral shifting effects occurring in the SOA under high-power clock injection within an extremely large duty cycle. In comparison with conventional approaches, the dark-optical-comb injected single-SOA-based RZ decision-gate exhibits distinguished features, including a simplified configuration, a noninverted polarity, a wavelength preserving operation, an ultralow duty cycle, a high ER, a wide dynamic range, and a large timing tolerance.

To realize the systematic performance of the proposed scheme, we have compared in Table I the characteristics parameters with those achieved by previous configurations, such as the power penalty $(P)$, the wavelength preserving capability $(\Delta \lambda)$, the duty cycle of the decision-gated RZ data $(\Delta \tau / \tau)$, the ON/OFF ER of the decision-gated RZ data $(R)$, the dynamic range of the input data $(\Delta P)$, and the timing tolerance in unit of duty cycle between the input data and the injected dark-opticalcomb clock for decision-gating $(\Delta T)$. It is observed that the decision-gated RZ data obtained from our approach has reached a relatively small duty cycle with high ER and negative receiving power penalty among all reported schemes, while providing a maximum timing tolerance and a highest dynamic range to the input data stream. Although the proposed system employs the XGM technique, it is a wavelength preserving operation as compared to previous approaches using wavelength converted operation. The dynamic range and timing tolerance of up to $14 \mathrm{~dB}$ and 34\% duty cycle, respectively, are the best records. In brief, the single SOA-based all-optical decision-gate by reshaped-optical-clock (dark-optical-comb) injection can precisely recover the distorted RZ data to reach good signal quality, even at a relatively low level of the input data. The dynamical $3 \mathrm{R}$ regeneration is suitable for the retrieval burst-mode dynamical signals. Such a new decision-gate effectively enlarges the dynamical range and the sensitivity of receivers, which is efficient for the enhancement of the transmitting distance of $10-\mathrm{Gb} / \mathrm{s}$ fiber-optics systems.

\section{CONCLUSION}

An all-optical OC-192 RZ decision-gate based on backward dark-optical-comb injected SOA is implemented, which simultaneously achieves reshaping and reamplifying of a degraded 
PRBS RZ data stream at $10 \mathrm{~Gb} / \mathrm{s}$. The scheme of our decision gate has the potential advantages of $10-\mathrm{Gb} / \mathrm{s}$ operation speed, simple implementation, larger dynamical range, and noninverted data priority. We experimentally and theoretically analyze the effect of the reshaped optical clock (i.e., the dark-optical-comb), injecting power and wavelength on the RZ-data reamplifying and reshaping to obtain the optimal ER of the decision-gated RZ pattern. Evaluation on the improved 3R regeneration performances, including the timing tolerance of $33.5 \mathrm{ps}$, the input dynamical tolerance of $15 \mathrm{~dB}$, and the ER of $14 \mathrm{~dB}$, are discussed. The SNR and the eye-diagram analyses at $10 \mathrm{~Gb} / \mathrm{s}$ reveals an improvement on the statistically distributed $Q$-factor from 6.0 to 8.1 , corresponding to an optimization on the reachable BER of the decision-gated RZ data stream from $1 \times 10^{-9}$ and $2 \times 10^{-16}$ under a receiving power of $-6 \mathrm{dBm}$. After propagating through a $25-\mathrm{km}$ SMF spool, the dispersion tolerance is evaluated, and the power penalty can be reduced from 3.2 to $0.7 \mathrm{~dB}$. Such a decision gate exhibits a threshold injection power of about $7 \mathrm{dBm}$ for the backward injected dark-optical-comb clock signal at an RZ data speed of up to $10 \mathrm{~Gb} / \mathrm{s}$.

\section{REFERENCES}

[1] F. Ratovelomanana, N. Vodjdani, A. Enard, G. Glastre, D. Rondi, R. Blondeau, A. Dupas, L. Billes, and J. C. Simon, "Regeneration improvement in all-optical wavelength converter, based on a Mach-Zehnder interferometer, by means of phase-shifter section," Electron. Lett., vol. 33, no. 19, pp. 1629-1630, Sep. 1997.

[2] W. Pender, T. Widdowson, and A. D. Ellis, "Error free operation of a $40 \mathrm{~Gb} / \mathrm{s}$ all-optical regenerator," Electron. Lett., vol. 32, no. 6, pp. 567-569, Mar. 1996.

[3] W. Pieper, K. Weich, R. Ludwig, E. Patzak, and H. G. Weber, "Alloptical polarisation and wavelength independent $3 \mathrm{R}$ signal regenerator," Electron. Lett., vol. 32, no. 14, pp. 1316-1318, Jul. 1996.

[4] A. E. Kelly, I. D. Phillips, R. J. Manning, A. D. Ellis, D. Nesset, D. G. Moodie, and R. Kashyap, " $80 \mathrm{~Gb} / \mathrm{s}$ all-optical regenerative wavelength conversion using semiconductor optical amplifier based interferometer," Electron. Lett., vol. 35, no. 17, pp. 1477-1478, Aug. 1999.

[5] C. Bornholdt, J. Slovak, and B. Sartorius, "Semiconductor-based alloptical 3R regenerator demonstrated at $40 \mathrm{~Gb} / \mathrm{s}$," Electron. Lett., vol. 40, no. 3, pp. 192-194, Feb. 2004.

[6] K. S. Jepsen, A. Buxens, A. T. Clausen, H. N. Poulsen, B. Mikkelsen, and K. E. Stubkjaer, " $20 \mathrm{~Gb} / \mathrm{s}$ optical 3R regeneration using polarisationindependent monolithically integrated Michelson interferometer," Electron. Lett., vol. 34, no. 5, pp. 472-474, Mar. 1998.

[7] S. Zischer, M. Dulk, E. Gamper, W. Vogt, E. Gini, H. Melchior, W. Hunziker, D. Nesset, and A. D. Ellis, "Optical 3R regenerator for $40 \mathrm{~Gb} / \mathrm{s}$ networks," Electron. Lett., vol. 35, no. 23, pp. 2047-2049, Nov. 1999.

[8] M. Eiselt, W. Pieper, and H. G. Weber, "Decision-gate for all-optical data retiming using a semiconductor laser amplifier in a loop mirror configuration," Electron. Lett., vol. 29, no. 1, pp. 107-109, Jan. 1993.

[9] A. Buxens, H. N. Poulsen, A. T. Clausen, and P. Jeppesen, "All-optical OTDM-to-WDM signal-format translation and OTDM add-drop functionality using bidirectional four wave mixing in semiconductor optical amplifier," Electron. Lett., vol. 36, no. 2, pp. 156-158, Jan. 2000.

[10] Y. Wang, J. Yu, L. Zhu, Y. Zhang, and Y. Enze, "Experimental study on $40 \mathrm{~Gb} / \mathrm{s}$ all-optical optical decision based on cross-gain modulation in SOA," Proc. SPIE, vol. 6021, pp. 60 210B-1-60 210B-8, 2005.

[11] L. Zhu, J. Yu, A. Zhang, Y. Wang, Y. Zhang, and Y. Enze, " $40 \mathrm{~Gb} / \mathrm{s}$ all-optical 3R regeneration based on F-P filter and SOA," Proc. SPIE, vol. 6021, pp. 60210I-1-60210I-8, 2005.

[12] G.-R. Lin, Y.-S. Liao, and G.-Q. Xia, "Dynamics of optical backward injection induced gain-depletion modulation and mode-locking in semiconductor optical amplifier fiber laser," Opt. Express, vol. 12, no. 10, pp. 2018-2026, May 2004.

[13] G.-R. Lin and I.-H. Chiu, "Femtosecond compression and wavelength tuning of backward-optical-injection harmonic-mode-locked SOA fiber laser at $10 \mathrm{GHz}$," Opt. Express, vol. 13, no. 22, pp. 8772-8780, Oct. 2005.
[14] G.-R. Lin, K.-C. Yu, and Y.-C. Chang, "10 Gb/s all-optical NRZ-to-RZ data format conversion based on a backward dark-optical-comb injected semiconductor optical amplifier," Opt. Lett., vol. 31, no. 10, pp. 13761378, May 2006

[15] I. D. Henning, M. J. Adams, and J. V. Collins, "Performance predictions from a new optical amplifier model," IEEE J. Quantum Electron., vol. QE-21, no. 6, pp. 609-613, Jun. 1985.

[16] G. P. Agrawal and N. A. Olsson, "Self phase modulation and spectral broadening of optical pulses in semiconductor laser amplifiers," IEEE $J$. Quantum Electron., vol. 25, no. 11, pp. 2297-2306, Nov. 1989.

[17] N. S. Bergano, F. W. Kerfoot, and C. R. Davidsion, "Margin measurements in optical amplifier system," IEEE Photon. Technol. Lett., vol. 5, no. 3, pp. 304-306, Mar. 1992.

[18] S. Sangbae, A. Byung-gu, C. Mungweon, C. Seongdae, K. Daejeong, and P. Youngil, "Optics layer protection of Gigabit-Ethernet system by monitoring optical signal quality," Electron. Lett., vol. 38, no. 19, pp. 11181119, Sep. 2002.

[19] J. M. Wiesenfeld, B. Glance, J. S. Perino, and A. H. Gnauck, "Wavelength conversion at $10 \mathrm{~Gb} / \mathrm{s}$ using a semiconductor optical amplifier," IEEE Photon. Technol. Lett., vol. 5, no. 11, pp. 1300-1303, Nov. 1993.

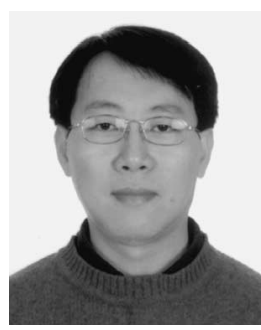

Gong-Ru Lin (M'93-SM'04) received the Ph.D. degree of electrooptical engineering from National Chiao Tung University, Hsinchu, Taiwan, R.O.C., in 1996.

$\mathrm{He}$ is currently a Professor and Director of the Laboratory of Fiber Laser Communications and Si Nano-Photonics, Graduate Institute of ElectroOptical Engineering and Department of Electrical Engineering, National Taiwan University, Taipei, Taiwan. He has authored or coauthored more than 100 papers in international journals and over 150 papers in international conference publications. His research interests include femtosecond fiber lasers, nanocrystallite Si photonics, all-optical data processing, and millimeter-wave photonic phase-locked loops.

Dr. Lin is a member of Optical Society of America and The International Society for Optical Engineers (SPIE). He is currently the Vice Chairs of SPIE Taiwan Chapter and the IEEE/Lasers and Electro-Optics Society Taipei Chapter.

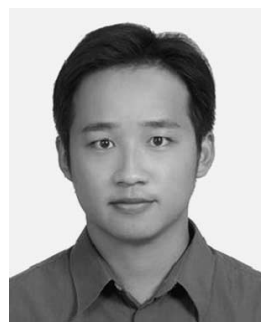

Kun-Chieh Yu was born in Taoyuan, Taiwan, R.O.C., in 1982. He received the B.S. degree from the Department of Electro-Optical Engineering, National Taipei University of Technology, Taipei, Taiwan, in 2005. He is currently working toward the M.S. degree at the Department of Photonics and Institute of Electro-Optical Engineering, National Chiao Tung University, Hsinchu, Taiwan.

Ci-Ling Pan, photograph and biography not available at the time of publication.

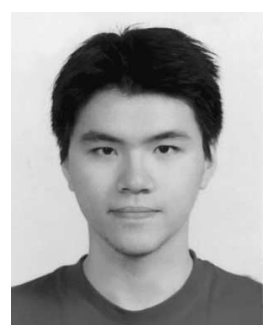

Yu-Sheng Liao was born in Taipei, Taiwan, R.O.C., in 1978. He received the B.S. dual degrees in applied mathematics and electrical engineering from National Chiao Tung University, Hsinchu, Taiwan, in 2002, where he is currently working toward the $\mathrm{Ph} . \mathrm{D}$. degree with the Department of Photonics and Institute of Electro-Optical Engineering.

His research interests are in all optical data processing and wavelength-division-multiplexing passive-optical-network systems. He has authored or coauthored more than seven papers in international periodicals and over 20 papers in international conference publications 